\title{
TTR
}

Traduction, terminologie, re?daction

\section{Hugo, père et fils, Shakespeare et la traduction}

\section{Nicole Mallet}

Volume 6, numéro 1, 1er semestre 1993

\section{L'Histoire en traduction}

URI : https://id.erudit.org/iderudit/037140ar

DOI : https://doi.org/10.7202/037140ar

Aller au sommaire du numéro

\section{Éditeur(s)}

Association canadienne de traductologie

\section{ISSN}

0835-8443 (imprimé)

1708-2188 (numérique)

Découvrir la revue

Citer cet article

Mallet, N. (1993). Hugo, père et fils, Shakespeare et la traduction. TTR, 6(1),

113-130. https://doi.org/10.7202/037140ar d'utilisation que vous pouvez consulter en ligne.

https://apropos.erudit.org/fr/usagers/politique-dutilisation/ 


\section{Hugo, père et fils, Shakespeare et la traduction}

\section{Nicole Mallet}

Victor Hugo rapporte la conversation suivante dans les premières pages de son William Shakespeare. La scène est à Jersey, très exactement à "Marine-Terrace», la première résidence des Hugo dans les îles anglo-normandes, au début des années 1850:

Un matin de la fin de novembre, deux des habitants du lieu, le père et le plus jeune des fils, étaient assis dans la salle basse. Ils se taisaient comme des naufragés qui pensent. [...]

Tous deux songeaient, absorbés peut-être par cette coïncidence $d^{\prime}$ un commencement $d^{\prime}$ hiver et $d^{\prime}$ un commencement d'exil.

Tout à coup le fils éleva la voix et interrogea le père:

- Que penses-tu de cet exil?

- Qu'il sera long.

- Comment comptes-tu le remplir?

Le père répondit:

- Je regarderai l'Océan.

Il y eut un silence. Le père reprit:

- Et toi?

- Moi, dit le fils, je traduirai Shakespeare. (Éd. Leuilliot, pp. 37-38)

Par delà la théâtralité hugolienne de cette évocation emblématique se profile une relation exceptionnelle à maints 
égards entre deux hommes hors du commun et qui gravite autour de la réception d'une œuvre et d'un auteur de génie que Victor Hugo nommera métaphoriquement un «homme océan». La question de François-Victor à son père devait conduire à l'éclosion d'un dialogue intellectuel prolongé et fécond qui allait doubler les liens affectifs réels des deux hommes d'une collaboration créatrice étonnante dont on n'a sans doute pas encore mesuré toute l'ampleur. Ce double projet démiurgique correspond aux quinze années qui vont de l'arrivée de la famille Hugo dans les îles Anglo-Normandes en 1851 à la parution du dernier volume de la traduction de Shakespeare, celui des apocryphes, par François-Victor Hugo en 1866. En fait, son ancrage dans l'histoire déborde en amont et en aval de ces limites temporelles dans la mesure où certaines des idées exprimées reprennent ou préfigurent certaines notions fondamentales qui se sont nuancées tout le long du parcours spirituel des deux hommes. On peut dire cependant que cet échange est concrétisé dans un ensemble textuel complexe dont l'unité et la cohérence sont assurés par le génie de Shakespeare et qui se présente comme suit:

A) Notes et commentaires de François-Victor Hugo traducteur, CEuvres complètes de W. Shakespeare (Paris, Pagnerre, in- $8^{\circ}$. B.N. yk. 5406-5423. Le tome VIII manque. On peut le trouver dans la deuxième édition: yk. 2342).

I. Les Deux Hamlet. - 1859.

II. Féeries. [Le Songe d'une nuit d'été; la Tempête.] - 1859.

III. Les Tyrans. [Macbeth; le Roi Jean; Richard III]. - 1859.

IV. Les Jaloux. I: Troylus et Cressida; Beaucoup de bruit pour rien; le Conte d'hiver. - 1859.

V. Les Jaloux. II: Cymbeline; Othello. - 1860.

VI. Les Comédies de l'amour: la Sauvage apprivoisée; Tout est bien qui finit bien; Peines d'amour perdues. - 1860.

VII. Les Amants tragiques: Antoine et Cléopâtre; Roméo et Juliette. - 1860.

VIII. Les Amis: les Deux gentilshommes de Vérone; le Marchand de Venise; Comme il vous plaira. - 1860.

IX. La Famille: Coriolan; le Roi Lear. - 1861. 
X. La Société: Mesure pour Mesure; Timon d'Athènes; Jules César. - 1862.

XI. La Patrie. I: Richard II; Henry IV. - 1863.

XII. La Patrie II: Henry V. Henry VI, $1^{\text {àr }}$ partie. - 1863.

XIII. La Patrie III: Henry VI, $2^{e}$ et $3^{e}$ parties. Henry VIII. - 1863. XIV. Les Farces: les Joyeuses épouses de Winsor; la Comédie des erreurs; le Soir des Rois, ou ce que vous voudrez. 1864.

XV. Sonnets; Poèmes; Testament. - 1865.

À noter que le classement n'est pas chronologique mais thématique et révèle chez François-Victor Hugo une conscience remarquable de l'organisation structurelle de la création shakespearienne. C'est ainsi qu'il s'explique dans l'introduction du tome 3 (les Tyrans): "À mon avis, ces trois pièces, Macbeth, le Roi Jean, Richard III, sont les parties diverses d'une cuvre unique, les développements successifs de la même idée, les portions à la fois distinctes et inséparables d'une trilogie immense qui pourrait s'intituler Le Talion» (p. 57).

Chaque volume est composé des traductions accompagnées d'une introduction substantielle et de notes. La collection comporte aussi trois volumes d'apocryphes parmi lesquels seuls Périclès et Titus Andronicus sont reconnus comme faisant partie du canon shakespearien.

B) Autres éléments du paratexte de la traduction de François-Victor Hugo:

Hugo, François-Victor. «Observations générales sur cette traduction", op. cit, vol. I, 1859, pp. 5-8.

Hugo, Victor. «Préface pour la nouvelle traduction de Shakespeare par François-Victor Hugo», William Shakespeare, éd. Bernard Leuilliot (Paris, Flammarion, "Nouvelle bibliothèque romantique», 1973), pp. 337-348.

«Les Traducteurs», William Shakespeare, éd. Bernard Leuilliot (Paris, Flammarion, «Nouvelle bibliothèque romantique», 1973), pp. 423-449. 
. William Shakespeare, éd. Bernard Leuilliot (Paris, Flammarion, «Nouvelle bibliothèque romantique», 1973).

La "Préface pour la nouvelle traduction de Shakespeare" fut composée par Victor Hugo à la demande de l'éditeur parisien Pagnerre, à l'heure où se préparaient les célébrations du tricentenaire de la naissance de Shakespeare (1864). Elle ne paraîtra en fait qu'en 1865 avec le volume de la traduction qui contient les sonnets. Ce deuxième texte ne peut guère se lire indépendamment de l'essai de plus de trois cents pages intitulé William Shakespeare qui lui sert en quelque sorte de matrice démesurée et qui comporte lui-même toute une liasse de résidus que Hugo appelait les "copeaux" de son œuvre et qui sont connus sous le nom de Reliquat. Je centrerai mon analyse sur le double discours préfaciel du fils et du père en opérant à l'occasion quelques coups de sonde dans le reste du corpus afin de mieux faire ressortir l'orchestration étonnante de cet ample discours sur la traduction et ses rapports à l'histoire. Toutes mes citations de William Shakespeare et de ses textes annexes proviennent de l'édition de Bernard Leuilliot répertoriée plus haut dans l'énoncé du paratexte.

J'aimerais, à l'orée de cette enquête, rendre hommage à Clio en puisant dans l'un des textes annexes de William Shakespeare: "On peut dire de la traduction en elle-même, écrit Victor Hugo, ce que Cicéron dit de l'histoire: quoque modo scripta, placet», ce qui signifie, Hugo donne en note la traduction: «elle plaît, quelle que soit la façon dont elle est écrite» (p. 436). Ce n'est pas tant le jugement de valeur qu'il renferme qui m'intẻresse dans cet aphorisme que l'équation fondamentale entre l'histoire et la traduction qu'il pose et qui servira d'axe a ma réflexion.

Il est bien vrai que le XIXe siècle est à la croisée des chemins de l'histoire et de la traduction. Le siècle des grands historiens est aussi celui du renouveau théorique de la traduction, surtout dans sa première moitié comme vient de le montrer savamment Lieven D'hulst (1990). Les deux routes au vrai se confondent dans l'avènement d'une nouvelle discipline, 
que l'on adjoindra à notre époque aux sciences humaines, la littérature comparée. Certains historiens du XIX ${ }^{e}$ ont été aussi de grands découvreurs des littératures étrangères: les travaux de Guizot ou de Taine en sont des exemples pertinents pour ce qui est de la littérature anglaise. Le rôle des traductions dans cet élargissement spatial et temporel de l'acquis cosmopolite du Siècle des Lumières est un chapitre important de l'histoire des échanges littéraires et culturels internationaux; les enquêtes entreprises par les critiques se réclamant de l'approche polysystémique ont récemment rectifié les prises d'angle et nuancé les données de cette étude. L'examen minutieux, effectué sur ces bases théoriques renouvelées, du discours critique qui sous-tend cette orientation nouvelle du cours de l'histoire des idées et des mentalités est une plus sûre garantie d'appréhension et de compréhension du mouvement de ces idées. Ainsi, les deux ouvrages récents de Lieven D'hulst $(1987,1990)$ mettent bien en valeur cette approche qui considère l'institution littéraire comme un système sémiotique complexe prenant en compte des phénomènes culturels, des faits sociaux et idéologiques que l'histoire littéraire traditionnelle avait eu tendance à ignorer et envisageant leurs interférences de façon dynamique. C'est de ce point de vue que j'aborde le paratexte de la traduction de Shakespeare par François-Victor Hugo.

J'examinerai tout d'abord les «Observations générales sur cette traduction" placées par le jeune traducteur en tête du premier volume de sa traduction (pp. 5-8), quatre pages qui datent de 1859. Il importerait de lire ces "Observations..." en corrélation avec l'ensemble du discours préfaciel des diverses traductions de l'œuvre de Shakespeare tout au cours du siècle (celles de Guizot, Francisque Michel, Benjamin Laroche, pour les œuvres dramatiques complètes; celles partielles de Vigny, Émile Deschamps, Alexandre Dumas, George Sand), tout un ensemble, un sous-système qui serait un bon laboratoire pour faire la généalogie de la théorie de la traduction et de la montée de l'emprise de l'histoire sur les esprits du siècle. Il y aurait là matière à une étude en soi et c'est en connaissance de cause que je me limite au contenu de ces quatre pages. 
D'entrée de jeu, François-Victor insiste sur ce qu'il juge être la nouveauté de son entreprise traductrice: «cette traduction sera nouvelle, dit-il, par la forme. [...] elle est faite, non sur la traduction de Letourneur, mais sur le texte de Shakespeare.» ( $p$. 5 ), et il inscrit d'emblée son propos dans une perspective historico-politique. $S^{\prime}$ il lui est loisible d'innover, selon lui, c'est justement parce que les conjonctures socio-culturelles sont opportunes: «Disons-le hautement, poursuit-il, pour qu'une traduction littérale de Shakespeare fût possible, il fallait que le mouvement littéraire de 1830 eût vaincu, il fallait que la liberté qui avait triomphé en politique, eût triomphé en littérature, il fallait que la langue nouvelle, la langue révolutionnaire, la langue du mot propre et de l'image, eût été définitivement créée» (p. 5). Lieven D'hulst (1987) a très bien relevé les incidences historiques du paradoxe de la littéralité comme garant d'adéquation dans le système-source et audace acceptable dans le système-cible (p. 19).

François-Victor Hugo situe donc résolument son travail philologique dans l'histoire des idées, il le justifie et l'explique à la fois par l'histoire du XIX' siècle et, ce faisant, il l'investit d'un pouvoir missionnaire qui l'inscrit dans l'ample mouvement de l'histoire de l'humanité. En effet, dans un élan mystique, il l'assimile à celui des exégètes des Écritures: «En comparant [..] la Bible shakespearienne aux reproductions qui en ont été faites plus tard, nous avons éprouvé en quelque sorte l'étonnement qu'avait ressenti Érasme en comparant l'Évangile grec à la Vulgate de saint Jérôme. Nous avons fait comme les protestants: plein d'une fervente admiration pour le texte sacré, nous en avons supprimé toutes les interpolations posthumes [...]» (p. 5).

$\mathrm{Du}$ reste, lorsqu'on parle du canon shakespearien, ce n'est pas un vain mot: le terme connote en vérité la qualité religieuse attribuée à la création du barde. François-Victor est retourné aux quartos lorsqu'ils existaient et, pour celles des pièces pour lesquelles il n'existe pas d'autres sources, au Folio de 1623 qui se trouvait chez un libraire de Guernesey. $A$ titre d'exemple et pour faire apprécier le sérieux du jeune chercheur, je relève sa remarque à propos de Roméo et Iuliette: 
Les diverses traductions de Roméo et Juliette qui jusqu'ici ont paru dans notre langue ont toutes été faites sur le texte inexact d'une édition toute moderne, publiée au siècle dernier par Steevens et Malone. À défaut d'autre qualité, la traduction que voici a du moins ce mérite tout nouveau de reproduire l'ceuvre de Shakespeare telle que l'auteur l'a écrite, et non telle que ses commentateurs l'ont forgée. Le texte que j'ai adopté est celui de l'édition in-quarto qui fut imprimée, en 1599, par Thomas Creed pour Cuthbert Burby et qui a servi de type aux éditions de 1609 et de 1623. (Note 34 , tome 7, p. 383 )

François-Victor Hugo avait commencé ses recherches dès 1853, allant régulièrement au British Museum. Peu de temps après son arrivée à Guernesey, il s'était épris d'une jeune Guernesiaise, Émilie de Putron qui «lui expliquait les nuances fines du vieil anglais», tandis que lui «lui apprenait les sources de Shakespeare" (Guille, 1950, p. 145). Du reste, il connaissait bien l'anglais qu'il avait appris sur les conseils de sa mère, alors que, jeune journaliste engagé, il avait été incarcéré à Paris pour avoir pris fait et cause contre Louis-Napoléon. Ce désir de retour au texte original le plus fiable (on sait qu'il n'existe aucun manuscrit de Shakespeare) est l'un de ses soucis majeurs et il y reviendra plusieurs fois scrupuleusement dans ses notes.

Double innovation de forme et d'esprit, donc, sensible dans ce qu'il considère "une autre curiosité de cette édition [...] [qui] sera de citer intégralement, dans des préfaces explicatives, les cuvres aujourd'hui oubliées qui ont été comme les esquisses des chefs-d'œuvre de Shakespeare» (p. 6). Une fois posé ce principe directeur archéologique, François-Victor ne reviendra plus sur les assises théoriques de son travail. Chacun des volumes qui construisent ce monument se compose du texte traduit encadré $d$ 'une abondante introduction et d'un appareil de notes non moins copieux. Sur le plan du principe, François-Victor n'inaugure pas totalement; Lieven D'hulst (1990), qui a étudié en détail la rhétorique préfacielle des traducteurs entre 1748 et 1847 , a constaté que «après 1815 , les commentaires sur la traduction [...] disparaissent simplement au profit de ce qui occupe 
davantage les esprits: la biographie de l'auteur, ou l'histoire sociale, littéraire ou culturelle du système-source» (p. 106).

Lorsqu'on se plonge dans l'encadrement textuel de la traduction de François-Victor Hugo, on est frappé par l'ampleur et la minutie des recherches entreprises: 1133 pages d'introductions (986 sans les apocryphes), 1185 pages de notes et appendices (1088 si l'on exclut les apocryphes). On se rend compte à quel point le jeune Hugo a accompli un travail de compilation attentif et exigeant, ce que son père exprimera avec ferveur dans sa «Préface»:

Un jeune homme s'est dévoué à ce vaste travail. À côté de cette première tâche, reproduire Shakespeare, il y en avait une deuxième, le commenter. L'une [....] exige un poète, l'autre un bénédictin. [...] Faisant cette tentative, il s'y est dépensé tout entier. Il a senti, en accomplissant cette tâche, la religion de construire un monument. Il y a consacré douze des plus belles années de la vie. (pp. 347-348)

Quelque vingt années plus tard, le poète Swinburne, grand admirateur de Victor Hugo, saluera ce travail avec la même déférence: «A monument of perfect scholarship, of indefatigable devotion and of literary genius». Il est vrai que le rythme des publications est essoufflant puisque les 18 volumes ont paru entre 1859 et 1866.

Lorsqu'on examine ce corpus critique, il se révèle à première lecture comme une somme de documentation historique relative au sujet ou au contexte de chacune des pièces traduites. Cependant, dans ce cas précis, c'est l'utilisation interprétative que le jeune traducteur en fait qui est originale. François-Victor ne se livre pas à une évocation anecdotique du contexte sociohistorique élisabéthain, ni non plus à une simple étude de sources. Il a tenté ardemment de reconstruire tout l'avant-texte de la création shakespearienne; il s'agit d'une enquête d'ordre épistémologique, sorte de prolégomène à une herméneutique qu'il juge nécessaire à une meilleure connaissance de Shakespeare. Pour ce faire, il n'a pas hésité à traduire des pans entiers des vieilles chroniques ou d'autres textes contemporains. 
C'est ainsi que pour le Roi Lear, par exemple, il a traduit du latin des extraits de la Chronique bretonne de Geoffroy de Monmouth; de l'anglais, de nombreux passages de l'Arcadie de Sir Philip Sydney et aussi de la Vraie Chronique du roi Leir, texte anonyme de 1605. Les critiques shakespeariens modernes ont beau jeu de condamner tous ces commentaires comme des élucubrations caduques; il n'empêche que la pratique est éminemment révélatrice pour nous d'un moment intéressant de l'histoire de la traduction. François-Victor Hugo était conscient de la portée et de la spécificité de son travail. Dans une lettre à son frère Charles, en 1868, il en parle comme de «l'édition-type pour les hommes d'étude et de cabinet» (Guille, p. 332). Il semble que pour lui la fidélité ne soit pas seulement affaire de littéralité, le respect de la lettre du texte étant garant d'exactitude; il importe qu'elle soit étayée par l'histoire qui lui donne sa dimension spirituelle. Fidélité = philologie + érudition ou, si l'on préfère, littéralité + histoire.

Il faudrait à ce stade ouvrir une vaste parenthèse pour retracer les grandes lignes de la philosophie de l'histoire qui va informer toute la créativité de Victor Hugo à partir de l'exil et que partage son fils. Je rappellerai pour mémoire qu'au moment où le fils amassait ses documents historiques et s'attelait à sa traduction, le père composait la première série de la Légende des siècles, cette ample fresque de l'histoire de l'humanité écoutée «aux portes de la légende», et qui ne visait nullement à une simple «reconstitution de l'histoire mais [à] une présentation du passé de l'homme (et de son avenir) à travers tout ce qui est l'ombre mythique des époques disparues", pour citer l'un de ses commentateurs contemporains (Halbwachs, 1969, p. XXVII).

Le fils modeste s'abritera derrière le père; l'originalité de cette édition, dira-t-il avec fierté à la fin de ses "Observations...», c'est d'offrir au public français «l'auteur de Ruy Blas commentant l'auteur d'Hamlet" (p. 8). ll n'en demeure pas moins qu'il ne faudrait pas mésestimer la part que François-Victor a prise dans l'élaboration de la pensée de son père, ne serait-ce qu'en lui procurant une connaissance textuelle de Shakespeare. Les aperçus fulgurants sur Hamlet, Macbeth, Othello et le Roi Lear qui sont 
parmi les pages les plus inspirées de l'essai rhapsodique sur William Shakespeare de Victor Hugo s'appuient sur une connaissance de la langue anglaise qu'il n'avait pas, qu'il n'a jamais eue et que seul l'entretien intellectuel et esthétique quotidien avec son fils cadet a pu lui donner.

Avant de s'exiler volontairement pour suivre son père, François-Victor Hugo avait été journaliste politique de métier, et un journaliste engagé puisque ses articles lui ont valu d'être arrêté et mis en prison en 1851. Dans ses chroniques de l'Événement, journal au titre évocateur qu'il avait fondé avec son frère Charles, il procédait à des analogies avec le théatre de Shakespeare: par exemple à la date du 30 novembre 1850, il tire des leçons de Roméo et Juliette pour interpréter la situation politique de son pays (Guille, p. 59). En 1854 dans l'Almanach de l'exil, il avait publié un extrait de sa traduction de Hamlet avec une série de réflexions sur «la démocratie dans Shakespeare» (rapporté par Guille, p. 95). Son intérêt pour Shakespeare s'avère inséparable de son souci de l'histoire, non pas uniquement en tant que chronique du passé mais de l'histoire en train de se faire. Deux ans avant la parution du premier tome de sa traduction, alors donc qu'il était en plein travail, il avait fait paraitre un volume intitulé la Normandie inconnue (1857); il y formule sa conception de l'histoire qui nous aide à saisir la vision unificatrice qui préside à son œuvre: «[l]'histoire, écrit-il, est un drame immense composé par un génie tout-puissant et joué par quatre personnages: la Papauté, la Féodalité, la Royauté, le Peuple. [...] trois actes ont été joués; le rideau se leve sur le quatrième.» (Cité par Guille, p. 116)

Il est aisé, certes, de percevoir dans ces affirmations des échos de la marche de l'Histoire chère à l'auteur de Cromwell ou des Misérables, mais il est permis de penser que cette vision, élaborée par Victor Hugo aux grandes heures de la révolution romantique, est devenue, durant l'exil, un chant à deux voix. Osmose, symbiose, nul ne saura jamais la subtilité de cet échange idéologique entre le père et le fils. Il ne s'agit plus alors de l'histoire événementielle mais de l'histoire au sens structural du terme, de l'histoire «de longue durée» pour reprendre la 
terminologie de Fernand Braudel, l'un des plus prestigieux représentants de l'École des Annales, disparu en 1985, un siècle après Victor Hugo.

A ce propos, il est pour le moins paradoxal que ce soit à partir de l'exil, donc lorsque tous deux se trouvaient éloignés de l'événement historique, en marge de l'histoire qui se fait, que les deux hommes aient approfondi leur méditation sur le sens de toute action humaine et se soient attachés à sonder une conception de l'histoire globale qui articule un discours visionnaire sur le temps. A la fin de sa "Préface», Victor Hugo écrira avec l'emphase émotive qui le caractérise:

Cette traduction de Shakespeare, c'est, en quelque sorte le portrait de l'Angleterre envoyé à la France. À une époque où l'on sent approcher l'heure auguste de l'embrassement des peuples, c'est presque un acte, et c'est plus qu'un fait littéraire. Il y a quelque chose de pieux et de touchant dans ce don qu'un français offre à sa patrie, d'où nous sommes absents, lui et moi, par notre volonté et avec douleur. ( $p$. 348)

Et c'est solennellement daté: «Hauteville-House. Mai 1864».

Dans une lettre adressée à son cousin Alfred Assouline, François-Victor commente en ces termes son propre travail: «Moi, j'ai entrepris une lutte colossale avec le grand ennemi et infatigable adversaire qui s'appelle le temps [...] la besogne $\mathrm{d}^{\prime}$ Hercule était une sinécure à côté de la mienne» ${ }^{1}$. Il ne faut pas seulement voir dans cette notation, me semble-t-il, une simple boutade découragée face à l'ampleur de la tâche entreprise mais sans doute aussi l'expression de la pensée qui la sous-tend et la soutient, une pensée qui s'inscrit dans le rythme du progrès de l'humanité et prend corps dans une vision mythique de l'histoire.

1. Victor Hugo intime (Marpon et Flammarion, 1885), p. 179; cité par Guille, p. 164. 
Les quelques rares extraits de sa correspondance personnelle sont du reste les seuls exemples tangibles que nous ayons de la conscience qu'avait cet homme humble de l'envergure méritoire de son cuvre. C'est à son père qu'il incombera de prendre la mesure du labeur accompli et de lui rendre hommage dans sa «Préface»: «J'attends avec impatience cet immense coup de trompette, écrira François-Victor à son père de Bruxelles le 9 avril 1865. J'ai mieux que la fanfare de la Renommée; J'ai la fanfare de la gloire» (Guille, p. 318). L'écriture de ces dix pages suit de près celle des quelque 375 pages du William Shakespeare que Victor Hugo avait dû extraire des arcanes de son esprit créateur avant d'être capable de rédiger la préface demandée dans les limites raisonnables voulues. Les hugoliens actuels les plus chevronnés reconnaissent William Shakespeare comme un essai fondamental pour la compréhension de la poétique de Victor Hugo. L'essai sur Shakespeare, terminé à la date, ô combien symbolique, du 2 décembre 1863, à Hauteville-House, "par tempête", fut mis en vente le 14 avril 1864 , en pleine célébration shakespearienne. La préface, rédigée en mai 1864, parut avec le tome XV de la traduction, sorte de préface en postface qui semble se jouer ainsi de la chronologie.

Il s'agit, certes, d'un texte de circonstance et comme toute préface sollicitée auprès de qui est déjà célèbre (Hugo est en pleine gloire et en pleine rentabilité des Misérables), elle a une fonction utilitaire, publicitaire et une rhétorique qui l'apparente à la littérature encomiastique. Il faut donc faire dans cet éloge la part de l'hyperbole obligée où se marient la démesure verbale native de l'écrivain et la partialité affective du père. Au vrai, cette double charge émotionnelle n'enlève rien à l'authenticité des idées exprimées. Victor Hugo situe d'emblée le débat au niveau culturel, voire politique et il ne craint pas de le clamer avec des accents polémiques: "Une traduction est presque toujours regardée tout d'abord par le peuple à qui on la donne comme une violence qu'on lui fait. Le gout bourgeois résiste à l'esprit universel» (p. 339). Il oriente donc nettement le geste et le produit du traducteur vers la société-cible, les place à un niveau qui transcende les frontières nationales. C'est en ce sens qu'il peut brandir le bonnet rouge dont il avait jadis coiffé le vieux 
dictionnaire pour se tourner vers l'aspect langagier de l'entreprise et affirmer:

Traduire un poëte étranger, c'est accroître la poésie nationale; $[. .$.$] Une langue dans laquelle on transvase de la$ sorte un autre idiome fait ce qu'elle peut pour refuser. Elle en sera fortifiée plus tard, en attendant elle s'indigne.[...] Quelle idée a-t-on de venir lui mêler dans le sang cette substance des autres peuples? [...] Elle hait cette nourriture. [...] Jupiter enfant recrachait le lait de la chèvre divine.» (p. 339).

Georges Mounin s'est interrogé sur les idées entretenues par Victor Hugo sur le langage et il fait remarquer à juste titre que l'écrivain, sensibilisé aux langues étrangères dès l'enfance, «est contemporain d'une révolution scientifique dans le domaine de l'étude du langage, [qu'il a] entrevu beaucoup plus de choses que la majorité de ses homologues sur ce point» (Mounin, 1969, p. VI). Si, avant l'exil, il reste malgré tout fidèle aux conceptions du XVIII siècle sur la langue - et Mounin renvoie à l'article «Langage» de la Grande Encyclopédie rédigé par Rivarol - , "[c]'est probablement dans William Shakespeare [...] qu'on trouve enfin les traces les plus nettes et les plus étendues d'un contact établi entre les conquêtes de la linguistique historique, et la culture personnelle la plus assimilée de Hugo» (p. XVII). Dans ce début de préface, Hugo va plus loin que les grammairiens ou les rhétoriciens qui l'ont précédé puisqu'il assigne aux traductions une fonction de transformation d'une langue en contexte. Il y a un deuxième présupposé théorique en corollaire à celui-ci et qui peut se résumer ainsi: les traducteurs de Shakespeare qui ont précédé François-Victor ont fait un travail médiocre, incomplet, inexact sur le plan de la qualité mais qu'on se doit de replacer dans le dynamisme de l'histoire de la traduction. Tout travail de pionnier est ingrat mais utile et irremplaçable. C'est dans cette optique historique qu'il faut lire la section suivante de la préface; Hugo s'arrête un temps sur Pierre Le Tourneur et affirme: «il faut de la bravoure pour être Le Tourneur» (p. 340), c'est-a-dire pour avoir osé s'attaquer au «barbare de génie» fustigé par Voltaire, pour avoir eu l'audace de faire une infraction au canon en se risquant à le traduire, dût-on pour ce faire l'édulcorer, le 
mutiler pour pouvoir le naturaliser et ainsi «plaire à sa nation» comme il le dira dans sa préface aux Nuits de Young):

Ce digne Le Tourneur, couronné à Montauban et à Besançon, lauréat académique de province, uniquement occupé d'émousser Shakespeare lil dira plus loin qu'il «n'a pas traduit Shakespeare; il l'a, candidement, sans le vouloir, obéissant à son insu au goût hostile de son époque, parodié” (p. 342)] de lui ôter les reliefs et les angles, et de le faire passer, c'est-à-dire de le rendre passable, ce bonhomme, travailleur consiencieux, ayant pour tout horizon les quatre murs de son cabinet, doux comme une fille, incapable de fiel et de représailles, poli, timide, honnête, parlant bas, vécut toute sa vie sous cette épithète, misérable, qui lui avait jetée l'éclatante voix de Voltaire, et mourut à cinquante-deux ans, étonné. (p. 341)

L'évocation de Letourneur est une vignette à valeur d'antiphrase, certes, dans l'économie générale de ce texte pragmatique. Elle offre à son auteur une superbe transition pour chanter les mérites de la supériorité du travail de son fils:

Le danger de traduire Shakespeare a disparu aujourd'hui, reprend-il. On n'est plus un ennemi public pour cela. Mais si le danger n'existe plus, la difficulté reste. (p. 342)

Suivent des lignes qui pourraient composer une véritable anthologie de citations sur ce qu'il nomme, dans le fragment réservé «les Traducteurs", la «traduction sans muselière»:

[I]ntroduire [Shakespeare] de plain-pied [...] l'avouer, l'afficher, le proclamer, le promulguer, être sa chair et ses os, prendre son empreinte, mouler sa forme, penser sa pensée, parler sa parole, répercuter Shakespeare de l'anglais en français, quelle entreprise! (p. 342)

Victor Hugo consacre ensuite une section à inventorier ce qui fait que «Shakespeare est un des poètes qui se défendent le plus contre le traducteur» (p. 343), un écrivain qui force à une «traction sur l'idiome» pour reprendre sa robuste formule des 
«Traducteurs». C'est là qu'il rassemble en deux pages ce qu'il avait déjà abondamment developpé dans ce fragment et qui témoigne d'une prescience remarquable de la démarche théorique des adeptes de la théorie linguistique de la traduction, comme l'a récemment montré avec pertinence Roger Lassalle (1985). L'analyse pénétrante que Lassalle en donne est à ma connaissance la seule étude à ce jour qui ait été consacrée à ce texte passionnant et mérite d'être signalée.

Shakespeare, nous dit encore Hugo, «échappe par l'idée, il échappe par l'expression. [...] Shakespeare échappe au traducteur par le style; il échappe aussi par la langue» (p. 343). «Rien n'est plus laborieux que de faire coïncider les deux idiomes" (p. 344). Dans le fragment des "Traducteurs», Hugo affirmait de façon elliptique, voire sibylline: «La question philologique n'est pas autre chose que la question métaphysique. Les traducteurs y jettent beaucoup de lumière» (p. 440). Il revient ici sur cette donnée idéologique:

Shakespeare résiste par le style; Shakespeare résiste par la langue. Est-ce là tout? non. Il résiste par le sens métaphysique; il résiste par le sens historique; il résiste par le sens légendaire. [...] Il est aussi sagace et singulier dans l'histoire. Il est on ne peut mieux renseigné dans la tradition et dans le conte. Quant à sa philosophie elle est étrange; elle tient de Montaigne par le doute, et d'Ezéchiel par la vision. (p. 344)

On voit à quel point cette préface est une distillation des idées fondamentales de Victor Hugo sur l'opération de traduction, sur la tâche et la mission du traducteur développées dans son William Shakespeare.

Pour Victor Hugo langue/histoire/métaphysique/ légende ne semblent être qu'une seule et même entité, les composantes indissociables d'une philosophie de la totalité, les hypostases d'une pensée qui est de l'ordre de la croyance mystique. Cet "homme siècle», comme on s'est plu naguère à 
l'appeler $^{2}$, ce visionnaire était possédé d'une foi dans le progrès de l'humanité, animé d'une conception mythique de l'histoire qui peut se lire comme un texte et c'est pourquoi il met en équation textualité et idéologie, traduction et histoire. A la section IV du livre III qui sert de conclusion à William Shakespeare («l'Histoire réelle - chacun remis à sa place») Hugo déplore la façon dont l'histoire a été écrite jusqu'alors; il appelle l'avènement d'une nouvelle Histoire ( Ill est temps que les génies passent devant les héros») et il écrit:

Un siècle est une formule; une époque est une pensée exprimée. Après quoi la civilisation passe à une autre. La civilisation a des phrases. Ces phrases sont les siècles. Elle ne dit pas ici ce qu'elle dit là. Mais ces phrases mystérieuses s'enchaînent; la logique - le logos - est dedans, et leur série constitue le progrès. Toutes ces phrases, expression d'un idée unique, l'idée divine, écrivent lentement le mot Fraternité. (p. 328)

Mû par cette vision fondamentale, il pourra écrire dans la "Préface»: "Comme la question biblique, comme la question homérique, comme la question dantesque, la question shakespearienne existe. L'étude de cette question est préalable à la traduction. Il faut d'abord se mettre au fait de Shakespeare» (p. 345). Il entend par là un travail de recherche sur les sources possibles d'inspiration du dramaturge non pas tant par éthique professionnelle, que par amour de la vérité car, «[l]e vrai traducteur doit faire effort pour lire tout ce que Shakespeare a lu. Il y a là pour le songeur des sources, et pour le piocheur des trouvailles» (p. 345). Voilà donc posée la justification épistémologique de l'appareil critique édifié par François-Victor pour escorter sa traduction. Le retour en arrière doit être une saisie imaginative de l'emprise du passé sur le présent et l'avenir: «Shakespeare était un puissant assimilateur. Il s'amalgamait le passé. [...] Une insufflation sortait pour lui du lourd tas des chroniques. De ces in-folio il dégageait des fantômes.» (p. 346)

2. Henri Meschonnic, "Victor Hugo en homme siècle", Romantisme, 60 (1988), pp. 57-70. 
"[Q]uand on s'est fait en soi un fac-simile du passé tel qu'il le voyait, [...] quand on a digéré tous ses faits, toute cette histoire, toutes ces fables, toute cette philosophie, quand on a gravi cet escalier de volumes, on a pour récompense cette nuée d'ombres divines au-dessus de sa tête.» (p. 347) Le cheminement du traducteur est ardu mais exaltant: «C'est le chemin de pierres de ce paradis.» (p. 346)

En guise de conclusion, j'insisterai sur la conception originale de la traduction partagée par les deux Hugo, le père comme le fils, qui envisagent la traduction de Shakespeare comme un système textuel composé du texte poétique (théâtre, sonnets) traduit et des présentations et commentaires historiques. Pour eux, le traducteur c'est le linguiste et l'historien confondus œuvrant en vue d'un seul et même acte de communication. Je cite le père qui saluait ainsi le travail de son fils: "CEuvre de critique, œuvre de philologie, œuvre de philosophie, œuvre d'histoire, qui côtoie et corrobore la traduction.» (p. 347) L'histoire est à la fois caution et contre-épreuve de la littérature traduite ou, pour citer une fois de plus Victor Hugo, "Le commentaire couche Shakespeare sur la table d'autopsie, la traduction le remet debout» (p. 347); ce monument est bien «Iplour ceux qui veulent tout Shakespeare dans Shakespeare» (p. 347). Il n'est pas excessif de lire le contenu de ce double discours préfaciel comme un double questionnement portant sur la place de la traduction dans l'histoire et sur celle de l'histoire dans la traduction tout aussi bien, démarche qui traduit un double défi partagé à l'espace et au temps.

Université de l'Alberta

\section{Références}

BASSNETT, Susan et André LEFEVERE, dir. (1990). Translation, History and Culture. London and New York, Pinter Publishers. 
GUILLE, Francis Vernor (1950). François-Victor Hugo et son œuvre. Paris, Nizet.

HALBWACHS, Pierre (1969). «Le Poète de l'histoire. II. La pensée de l'exil", Victor Hugo, CEuvres Complètes. Edition chronologique, Club Français du livre, tome $X$ (18561860), pp. XXIII-XLVIII.

HULST, Lieven D' (1987). L'Évolution de la poésie en France (17801830). Leuven University Press.

(1990). Cent ans de Traduction en France (1748-1847). Presses universitaires de Lille.

LARTHOMAS, Pierre (1982). «Hugo linguiste», Le Génie de la forme, Mélanges de langue et littérature offerts à Jean Mourot. Presses universitaires de Nancy, pp. 409-417.

LASSALLE, Roger (1985). «Victor Hugo et l’idéologie poétique de la traduction", Idéologies hugoliennes. Ed. Serres, pp. 181189.

LEFEVERE, André (1991). "Translation and Comparative Literature. The Search for the Center», TTR, IV(1), 1st semester, pp. 129-144.

MOUNIN, Georges (1969). «Victor Hugo et le langage», Victor Hugo, CEuvres Complètes, Édition chronologique, Club Français du livre, tome $X(1856-1860), p p$. I-XXI. 